\title{
In Vitro and In Vivo Availability of Some Commercial Prednisolone Tablets
}

\author{
Timothy J. Sullivan, ${ }^{1}$ Roger G. Stoll, ${ }^{1.2}$ Ermelinda Sakmar, ${ }^{1}$ \\ Donald C. Blair, ${ }^{1}$ and John G. Wagner ${ }^{1.3}$
}

Received Aug. 27, 1973--Final Nov. 15, 1973

\begin{abstract}
The in vitro dissolution rates of prednisolone from five commercially available 5-mg prednisolone tablets were determined in distilled water. The dissolution studies were repeated on the fastest-and slowest-dissolving brands using $0.01 \%$ polysorbate 80 in $0.1 \mathrm{~N} \mathrm{HCl}$ as the dissolution medium. A two-way crossover bioavailability study was performed in 12 human male adult volunteers comparing the fastest-and slowest-dissolving brands. The plasna sumples were assayed for prednisolone by a radioimmunoassay method. Statistical analysis of the data for the two brands showed no significant differences between average plasma levels of prednisolone at any sampling time. The results suggest that on the average the in vivo rates of dissolution of the two brands were essentially the same, and in vitro dissolution in $0.01 \%$ polysorbate $80-0.1 \mathrm{~N} \mathrm{HCl}$ medium was better correlated with these in vivo results than dissolution in water.
\end{abstract}

KEY WORDS: bioavaila bility; prednisolone tablets; in vivo-in vitro bioavaila bility correlations ; prednisolone availability; radioimmunoassay for prednisolone.

\section{INTRODUCTION}

Recently, the Ad Hoc Committee on Drug Product Selection of the Academy of General Practice of Pharmacy and the Academy of Pharmaceutical Sciences has included prednisolone on its list of drugs with high risk potential for therapeutic inequivalence due to differences in bioavailability (1). Previous studies in man $(2,3)$ required that large doses of prednisolone be given due to the lack of sensitivity in the existing assay methods. The development of the radioimmunoassay for this steroid has allowed the

Supported by Contract CPF69-22, Food and Drug Administration, Washington, D.C, and in part by Public Health Service Grant 5-P11-GM15559.

${ }^{1}$ College of Pharmacy and Upjohn Center for Clinical Pharmacology, The University of Michigan, Ann Arbor, Michigan 48104.

${ }^{2}$ Present address : Bioavailability Unit, The Upjohn Company, Kalamazoo, Michigan.

${ }^{3}$ Address reprint requests to Dr. John G. Wagner, Upjohn Center for Clinical Pharmacology, The University of Michigan, Ann Arbor, Michigan 48104. 
determination of plasma levels after the administration of a single, low dose of prednisolone.

This study employs the radioimmunoassay in the determination of the bioavailability of two brands of prednisolone.

\section{EXPERIMENTAL}

\section{In Vitro Rate of Dissolution Studies}

The dissolution apparatus consisted of a three-necked round-bottom $1000-\mathrm{ml}$ flask suspended in a constant-temperature bath maintained at $37 \pm 1^{\circ} \mathrm{C}$. The dissolution medium was stirred at $50 \pm 3 \mathrm{rpm}$ with a stirrer blade described by Wagner (4) using a standard servodyne power drive system. Fluid was continuously circulated through a small syringe barrel fitted with glass wool and small glass beads (one end of the barrel being immersed in the dissolution medium) into $0.29 \mathrm{~cm}$ ID silicone tubing, through a quartz flow cell in a Beckman DU spectrometer, then through a variable-speed Masterflex pump ${ }^{4}$ and back into the dissolution flask. The dissolution medium was circulated at a rate of $70 \mathrm{ml} / \mathrm{min}$. A V-shaped glass tube filled partially with glass beads was placed in the silicone tubing line to act as a debubbling device. A $10 \mathrm{~mm}$ path length flow cell was employed. The absorbance of the filtered dissolution medium was read continuously on a Gilford 2400 spectrophotometer chart recorder at $248 \mathrm{~nm}$.

Before each experimental run, the entire system was allowed to come to equilibrium. At zero time, a single tablet was dropped through one of the side openings of the three-necked dissolution flask so that it ultimately rested at the bottom of the flask beneath the stirring blade. The absorbance was read continuously until greater than $75 \%$ of the prednisolone had dissolved, at which time the circulation of dissolution medium was stopped and the stirring speed was increased to $200 \mathrm{rpm}$. After $30 \mathrm{~min}$, the stirring speed was reduced to $50 \mathrm{rpm}$ and the circulation of fluid through the cell restored. This was repeated until a constant absorbance value was obtained.

Deionized water was used as the dissolution medium in the first series of dissolution studies. A second series was performed using $0.01 \%$ polysorbate 80 in $0.1 \mathrm{~N}$ hydrochloric acid as the dissolution medium (5). Tablets $\mathrm{A}, \mathrm{B}, \mathrm{C}, \mathrm{D}$, and $\mathrm{E}^{5}$ were used in the first dissolution study, but only tablets $A$ and $B$ were studied in the second.

\footnotetext{
${ }^{4}$ Cole-Parmer, Chicago, Ill.

${ }^{5}$ Tablet A was Meticortelone, $5 \mathrm{mg}$ (Schering Corp.), Lot No. 1ACC1P35944; tablet B was Sterane, $5 \mathrm{mg}$ (Pfizer), Lot No. 14424; tablet C was Ulacort, $5 \mathrm{mg}$ (Fellows Testagar), Lot No. 71628; tablet D was Delta-Cortef, $5 \mathrm{mg}$ (Upjohn), Lot No. 301AR-A2; and tablet E was Prednis, $5 \mathrm{mg}$ (USV Pharmaceutical Corp.), Lot No. 50492.
} 
Prednisolone powder ${ }^{6}$ was used to calibrate the recorder. The absorbance value, corrected for baseline shifts, at "infinite" time was used as the value for $100 \%$ steroid in solution.

\section{Subjects}

Twelve adult male volunteers between the ages of 23 and 39 years, weighing between 144 and $219 \mathrm{lb}$, and in good health were selected. Each subject received a medical history and physical examination. Values for the following tests were required to be within the normal range: hematocrit, white count, differential, SGOT, alkaline phosphatase, total bilirubin, serum creatinine, plasma 17-OHCS, electrocardiogram, and chest X-ray.

Each subject selected received no barbiturates or other enzyme-inducing agents for a period of 30 days preceding the study and none concurrent with it. They received no other medication or alcoholic beverages for a period beginning 7 days preceding the study until completion of the study.

The subjects were ordered so that the average body weights of subjects $1,2,3,10,11$, and 12 were about the same as the average body weights of subjects $4,5,6,7,8$, and 9 .

\section{Protocol}

Subjects fasted overnight and for $4 \mathrm{hr}$ after administration of each dose of prednisolone. On the mornings when medication was administered, each subject drank 8 fluid ounces of water within the first hour after arising. No other food or beverage was taken until $4 \mathrm{hr}$ after dosing with prednisolone. From $4 \mathrm{hr}$ after dosing with prednisolone, food and beverage were taken ad libitum.

Fifty milliliters of whole blood was taken from a forearm vein just before dosing with prednisolone at 0 time, and $10 \mathrm{ml}$ of whole blood was taken at $0.25,0.5,1,2,3,4,6,8,12$, and $24 \mathrm{hr}$ after dosing with prednisolone. The large initial blood sample was collected in a syringe and transferred to a heparinized tube. The $10-\mathrm{ml}$ blood samples were drawn into Vacutainer tubes containing heparin as the anticoagulant. Each sample of blood was centrifuged as soon as possible after collection, and the plasma was placed in a vial, stoppered, and labeled immediately. The plasma samples were then quick-frozen and stored in the frozen state until just prior to assay.

\section{Treatments and Schedules}

At 11:00 PM on day minus one (-1) of each treatment period, each subject took orally $1.0 \mathrm{mg}$ of dexamethasone ${ }^{7}$ to suppress endogenous secretion of hydrocortisone. 
Table I. Treatment Schedules

\begin{tabular}{lccc}
\hline Subjects & Group & $\begin{array}{c}\text { Phase I } \\
\text { (week 1) }\end{array}$ & $\begin{array}{c}\text { Phase II } \\
\text { (week 2) }\end{array}$ \\
\hline $1,2,3$ & I & A & B \\
$4,5,6$ & II & B & A \\
$7,8,9$ & III & B & A \\
$10,11,12$ & IV & A & B \\
\hline
\end{tabular}

At plus $8 \mathrm{hr}$ on day 1 (i.e., $8 \mathrm{hr}$ after prednisolone administration) of each treatment period, each subject took $0.5 \mathrm{mg}$ of dexamethasone ${ }^{8}$ orally.

On each phase of the crossover, each subject ingested orally $10 \mathrm{mg}$ of prednisolone as two 5-mg tablets of tablet $\mathrm{A}$ or tablet $\mathbf{B}$. The tablets were swallowed whole, not fractured or chewed before being swallowed. They were taken in the morning (about $8 \mathrm{AM}$ ) with 6 fluid ounces of water. The two doses were separated by a 1-week period.

Both brands chosen had been purchased on the open market by the Food and Drug Administration and the tablets had passed the official tests including average potency and tablet-to-tablet uniformity tests in the laboratories of the Food and Drug Administration.

The treatment schedules are shown in Table I.

\section{Assay of Plasma Samples}

Plasma samples were assayed using a radioimmumoassay for prednisolone. The antiserum was developed by Colburn and Buller (6), but their assay procedure has been greatly modified to suit our needs.

Each plasma sample had to be diluted with deionized water so that the binding of the tritiated prednisolone in the sample was in the most sensitive portion of the binding curve. This dilution factor was determined empirically. For this study, it was found that three distinct dilutions were required : 1:50 for the 1-, 2-, and 3-hr samples, $1: 25$ for the $\frac{1}{4}-\frac{1}{2}-, 4-, 6-$, and 8-hr samples, and 1:2 for the 12- and 24-hr samples. Individual samples often varied within this grouping.

The drug in $1 \mathrm{ml}$ of the appropriate sample dilution was extracted with $7 \mathrm{ml}$ of methylene chloride, shaken for $5 \mathrm{~min}$, and centrifuged for $5 \mathrm{~min}$. The aqueous layer was removed and a $5-\mathrm{ml}$ aliquot of the organic layer transferred to a disposable glass culture tube. The extract was evaporated to dryness at $37^{\circ} \mathrm{C}$ under a gentle stream of nitrogen gas.

Fifty microliters of absolute ethanol was allowed to wash down the sides of the dried culture tubes to aid solution. This was followed by $1.0 \mathrm{ml}$ of phosphate-buffered saline (7). This mixture was rapidly mixed on a

\footnotetext{
${ }^{8}$ Given as one tablet of Decadron (MSD), $0.5 \mathrm{mg}$.
} 
vortexer for $1 \mathrm{~min}$ to insure the complete dissolution of the steroid. Ten microliters of tracer stock solution and $10 \mu \mathrm{l}$ of undiluted antiserum were added and gently mixed. This solution was incubated for $2 \mathrm{hr}$ at $37^{\circ} \mathrm{C}$ in a shaking water bath.

At the end of the incubation period, the samples were immediately placed in an ice bath and $0.5 \mathrm{ml}$ of cold dextran-coated charcoal suspension was added. Dextran-coated charcoal suspension consisted of $125 \mathrm{mg}$ Dextran $\mathrm{T} 70^{9}$ and $5.0 \mathrm{~g}$ charcoal ${ }^{10}$ in $100 \mathrm{ml}$ of $p \mathrm{H} 7.4$ phosphate-buffered saline solution. After gentle mixing, this suspension was immediately centrifuged at $1500 \times \mathrm{g}$ for $20 \mathrm{~min}$ at $4^{\circ} \mathrm{C}$. The supernatant liquid was then added to $10 \mathrm{ml}$ Unogel ${ }^{11}$ liquid scintillation fluid. During the time between centrifugation and separation, the samples were kept in the ice bath. All samples were allowed to dark-adjust for at least $30 \mathrm{~min}$ before counting for $10 \mathrm{~min}$ in a Packard Tri-Carb liquid scintillation spectrophotometer. Each plasma sample was assayed in duplicate.

A calibration curve was prepared by assáying samples of diluted blank $(0 \mathrm{hr})$ plasma to which $1,2,4,6,10$, and in some cases $14 \mathrm{ng}$ of prednisolone had been added. In all cases, the dilutions of the blank plasma were the same as those of the samples and the blank plasma was that of the same subject whose prednisolone blood levels were being measured.

With each calibration curve, the following "controls" were included:

1. A blank to which no antiserum had been added. This measured the "background" radiation of the system.

2. A sample which contained only radioactive prednisolone (i.e., no "cold" drug). This measured the extent of the binding by the antiserum to the drug and was used as the " $100 \%$ bound" term.

3. A sample in which the radioactive drug had been added directly to the scintillation cocktail. This measured the total radioactivity initially in the system.

Since this assay measures the amount of tritiated prednisolone bound by the antiserum, the "percent bound" was calculated as follows:

$$
\text { Percent bound }=\frac{\text { sample reading }- \text { background reading }}{\text { total count }- \text { background reading }} \times 100
$$

A sample calibration curve is shown in Fig. 1. All calibration curves were fitted essentially perfectly by the NONLIN program on an IBM $360 / 67$ computer to a biexponential equation. The amount of drug in the experimental samples was then determined by an iterative program on a HewlettPackard 9100A calculator.

\footnotetext{
${ }^{9}$ Pharmacia Fine Chemicals, Uppsala, Sweden.

${ }^{10}$ Norit A, Sigma Chemical Co., St. Louis, Mo.

${ }^{11}$ Schwarz/Mann, Mountain View Ave., Orangeburg, N.Y.
} 


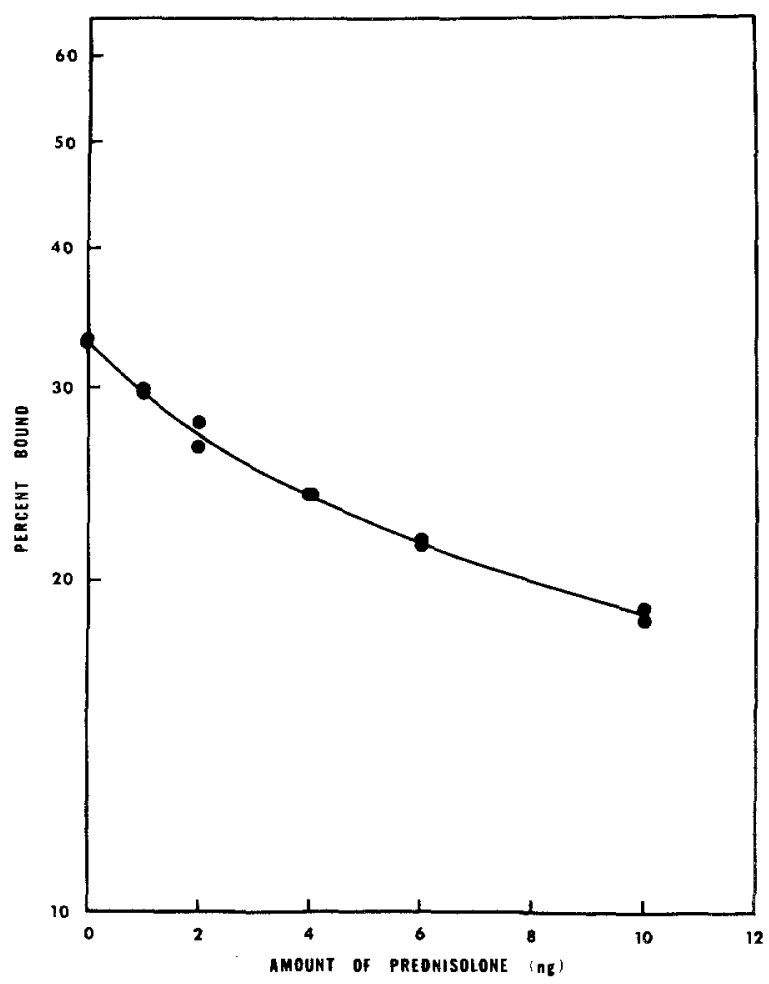

Fig. 1. Sample calibration curve for the radioimmunoassay of prednisolone.

\section{RESULTS AND DISCUSSION}

\section{In Vitro Rate of Dissolution Studies}

Figures 2 and 3 are plots of the dissolution data for tablets A, B, C, D, and $\mathrm{E}$ in deionized water. Each point corresponds to the average percent dissolved of four tablets of each lot. Clearly, tablets A and B represent the extremes in dissolution rates among the tablets tested. Therefore, tablets $\mathrm{A}$ and $\mathrm{B}$ were selected as the treatments for the in vivo bioavailability study. Tablet A is also the innovator's product.

The dissolution studies were repeated on tablets A and B using $0.01 \%$ polysorbate 80 in $0.1 \mathrm{~N}$ hydrochloric acid as the dissolution medium. Table II and Fig. 4 compare the effects of these two dissolution media on these tablets. Two way analysis of variance of $T_{25 \%}$ and $T_{50 \%}$ values indicated that the dissolution rates of the two brands were significantly different. The tablet dissolution rates in each dissolution medium were also significantly different $(p<0.001$ in all cases). 


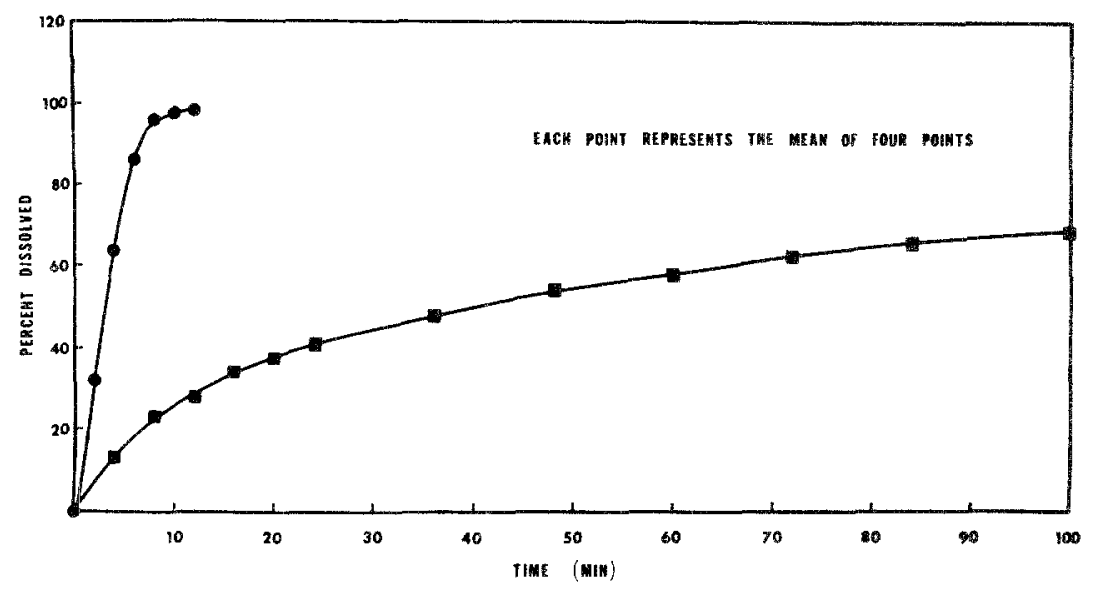

Fig. 2. In vitro dissolution of tablets $\mathrm{A}(\boldsymbol{)})$ and $\mathrm{B}(\mathbf{})$ in deionized water.

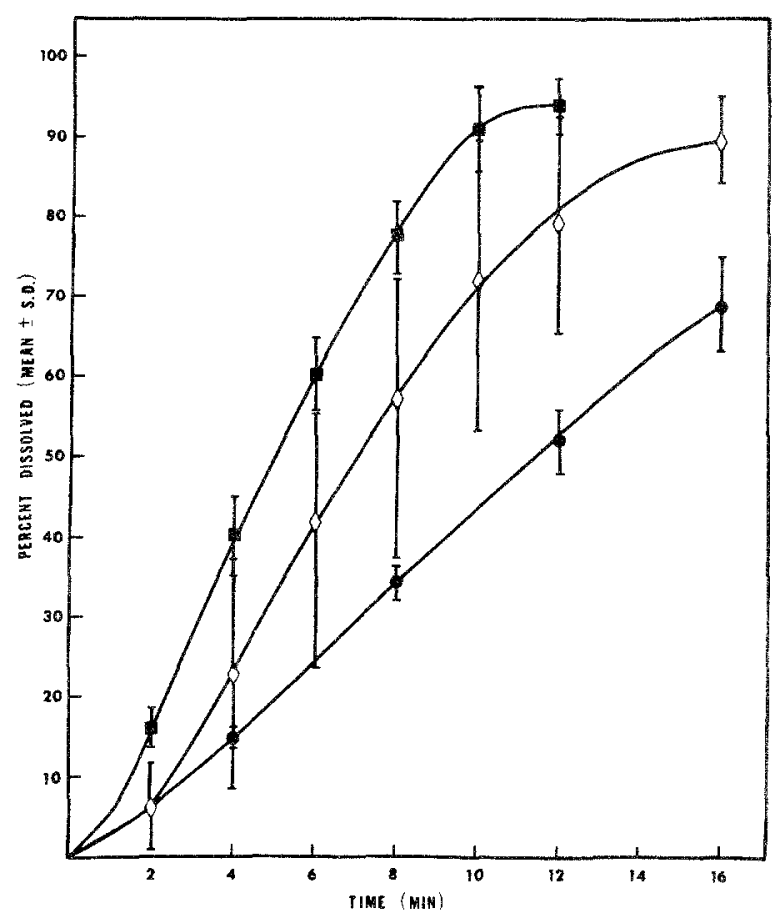

Fig. 3. In vitro dissolution of three commercial 5-mg prednisolone tablets in deionized water : tablet $\mathrm{C}(\mathbf{D})$, tablet $\mathrm{D}(\diamond)$, and tablet $\mathrm{E}(\mathbf{O})$. 
Table II. Summary of the Effect of the Dissolution Media on the Times Required to Dissolve 25,50 , and $75 \%$ of the Prednisolone in the Tablets ${ }^{a}$

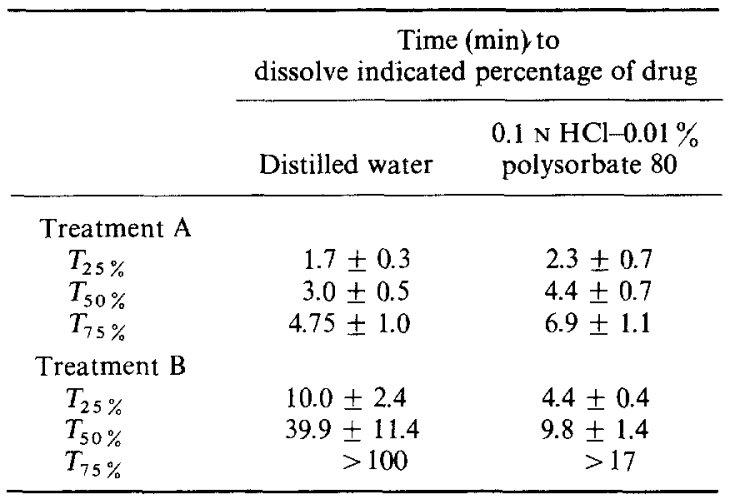

${ }^{a}$ Each value represents average of four trials $\pm S D$.

\section{Plasma Concentrations}

The cross-reactivity of prednisolone and cortisol with the antiserum was found to be similar to that reported by Colburn and Buller (6). In order to obtain a specific assay for prednisolone in plasma, it was necessary to

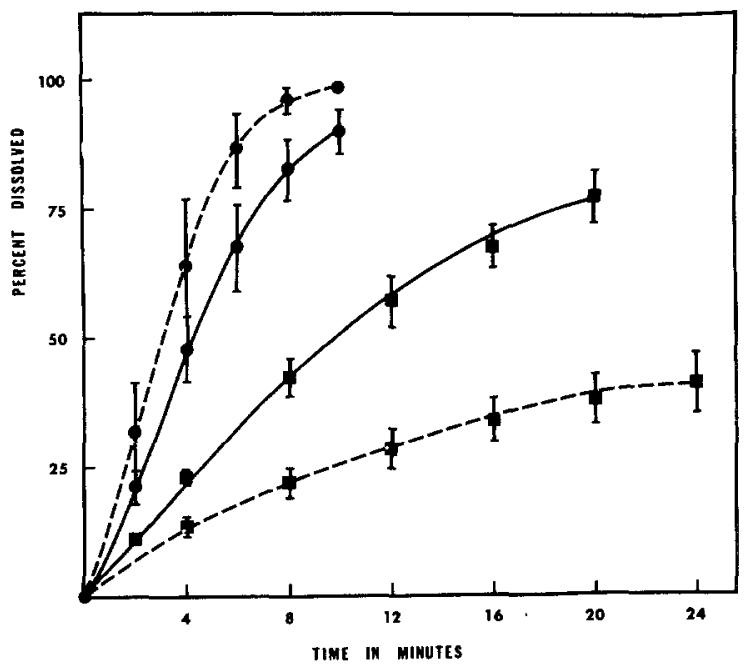

Fig. 4. Effect of dissolution media on the in vitro dissolution of tablets A (1) and B (C). Dotted lines indicate deionized water as the dissolution medium. Solid lines indicate the $0.01 \%$ polysorbate 80 in $0.1 \mathrm{~N} \mathrm{HCl}$ dissolution medium. 


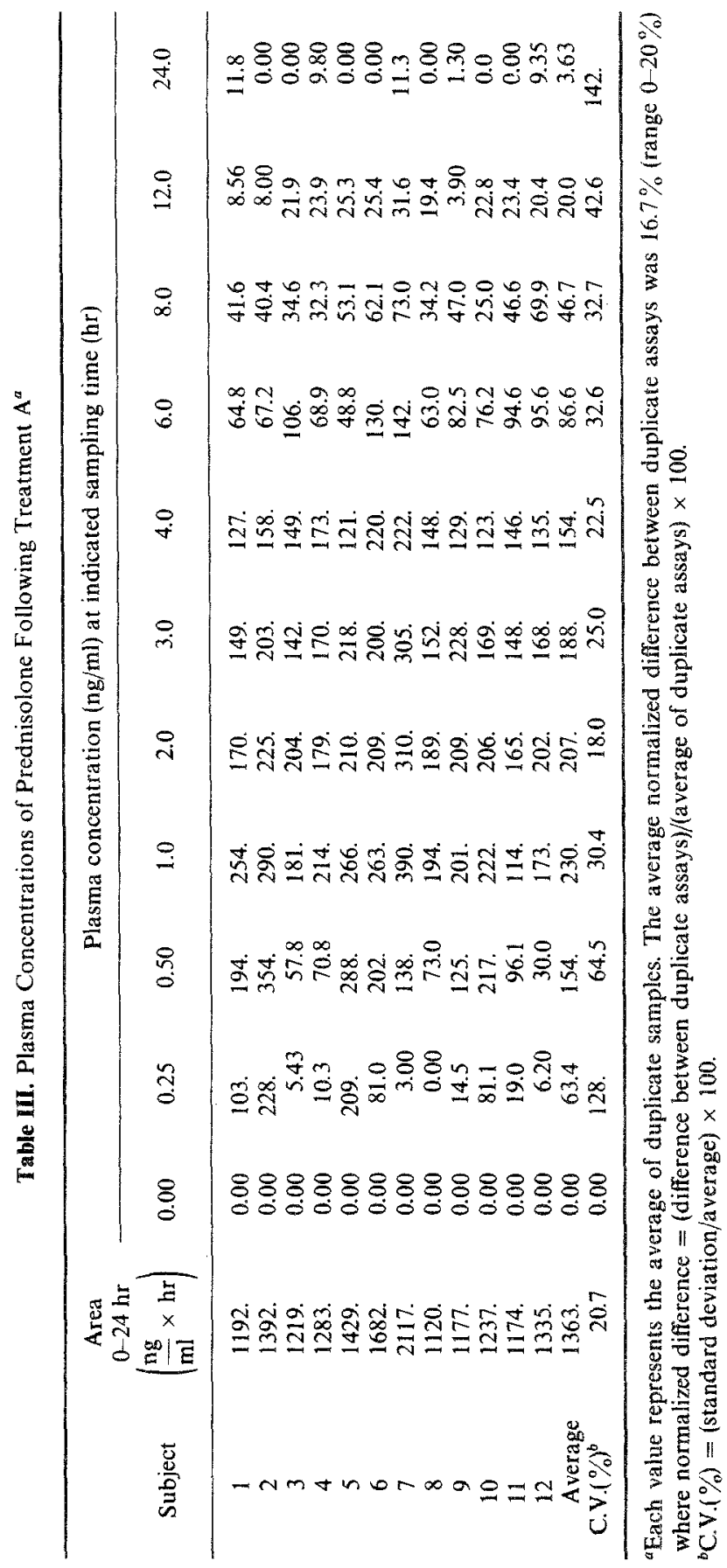




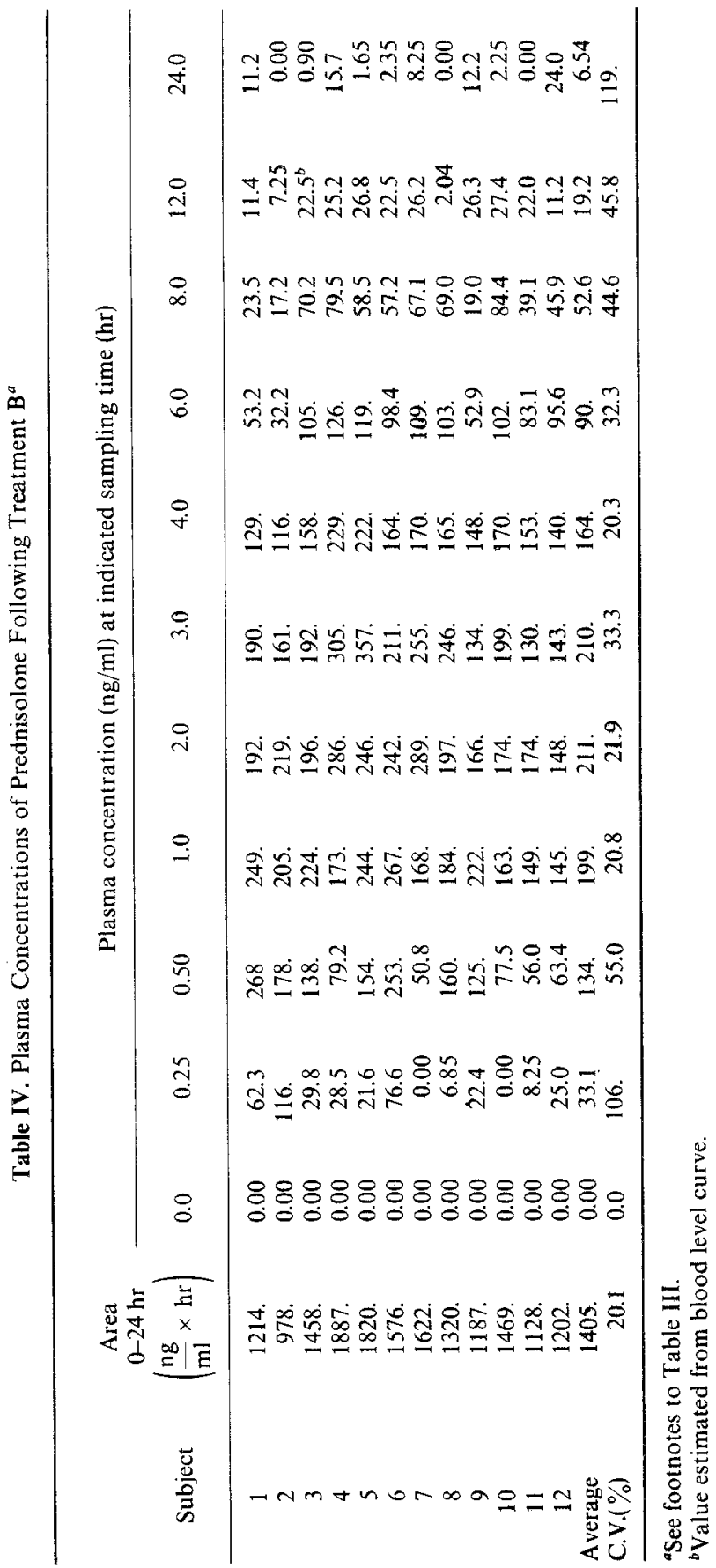


suppress the adrenal secretion of cortisol. This was accomplished by administering dexamethasone on the evening before and the afternoon of the day of the study. Dexamethasone, even in high concentrations, does not interfere with the binding of prednisolone to the antibody.

The plasma concentrations of prednisolone measured in each phase of the crossover study are shown in Tables III and IV. Each point is the average of duplicate samples whose values have been computed from the leastsquares fit of the calibration curve as described previously.

Table V summarizes the treatment averages for the prednisolone study. There are no significant differences $(p>0.05)$ between average plasma concentrations at each sampling time, in peak plasma concentrations, areas under the plasma-level curves (both $0-12 \mathrm{hr}$ and $0-24 \mathrm{hr}$ ), or half-lives. This indicates no significant difference in bioavailability of these two products.

\section{CONCLUSIONS}

The desirability of correlations of variables derived from in vitro dissolution rates with variables derived from in vivo testing in human

Table V. Summary of Results of 12-Subject Two-Way Crossover Prednisolone Study

\begin{tabular}{cccc}
\hline & \multicolumn{4}{c}{$\begin{array}{c}\text { Plasma concentrations }(\mathrm{ng} / \mathrm{ml}): \\
\text { averages for }\end{array}$} \\
\cline { 2 - 3 } Time $(\mathrm{hr})$ & Treatment A & Treatment B & p value \\
\hline 0.25 & 63.4 & 33.1 & $0.10<p<0.25$ \\
0.5 & 154 & 134 & $>0.25$ \\
1 & 230 & 199 & $0.10<p<0.25$ \\
3 & 207 & 211 & $>0.25$ \\
4 & 188 & 210 & $0.10<p<0.25$ \\
6 & 154 & 164 & $>0.25$ \\
8 & 86.6 & 90.0 & $>0.25$ \\
12 & 46.7 & 52.6 & $>0.25$ \\
24 & 20.0 & 19.2 & $>0.25$ \\
Peak plasma concentration ${ }^{b}(\mathrm{ng} / \mathrm{ml})$ & 3.63 & 6.54 & $0.05<p<0.10$ \\
Area 0-12 hr $\left(\frac{\mathrm{ng}}{\mathrm{ml}} \times \mathrm{hr}\right)$ & 248 & 243 & $>0.25$ \\
Area 0-24 hr $\left(\frac{\mathrm{ng}}{\mathrm{ml}} \times \mathrm{hr}\right)$ & 1224 & 1250 & $>0.25$ \\
Half-life $(\mathrm{hr})$ & 1363 & 1405 & $>0.25$ \\
\hline
\end{tabular}

"The $p$ value is the significance level of the treatment mean square from the analysis of variance for crossover design.

${ }^{b}$ The average of observed peak plasma levels of individual subjects. 
volunteers is well established in biopharmaceutics (8). In this study, the results suggest that the in vivo rates of dissolution of tablets $A$ and $B$ are on the average the same. Based on this information, the in vitro dissolution in $0.01 \%$ polysorbate $80-0.1 \mathrm{~N}$ hydrochloric acid medium correlates much better with the in vivo data than dissolution in water alone.

Finholt and Solvang (5) have used similar polysorbate 80-hydrochloric acid dissolution medium to explore the role of surface tension on dissolution rates. They have shown that dissolution of phenacetin and phenobarbital powder in these systems mimics dissolution in diluted gastric juice. The present data demonstrate the improvement of in vivo-in vitro correlations obtained by taking into account the surface tension of physiological fluids.

It is interesting that while the dissolution rate of tablet $\mathbf{B}$ increased dramatically in the second dissolution medium, the dissolution rate of tablet A was slightly slower. The dissolution of tablet A was still rapid and complete, so the cause of this phenomenon was not investigated. If some tableting ingredient is responsible for this change, it could significantly alter the in vivo dissolution of another tablet formulation while being undetectable with an in vitro dissolution test using water as the medium. Further investigation into this matter may be warranted.

Based on the data presented, it would be difficult to speculate on the bioavailability of tablets $\mathrm{C}, \mathrm{D}$, and $\mathrm{E}$ without further experimentation.

\section{ACKNOWLEDGMENTS}

The authors wish to thank Mr. W. A. Colburn and Dr. R. H. Buller, The Upjohn Company, for providing the antiserum and the radiolabeled prednisolone used in this study, and Dr. Carl M. Metzler, The Upjohn Company, for providing the digital computer program NONLIN.

\section{REFERENCES}

1. An annotated list of drugs with a potential for therapeutic inequivalence based on current evidence of drug product bioavailability inequivalence. J. Am. Pharm. Ass. NS13: 279-280 (1973).

2. J. P. Isbister, J. Speras, and A. W. Steinbeck. The absorption of prednisolone B.P. $5 \mathrm{mg}$. and $25 \mathrm{mg}$. tablets. Med. J. Aust. 1: 1135-1136 (1969).

3. P. F. D'Arcy, J. P. Griffin, J. S. Jenkins, W. F. Kirk, and A. W. C. Peacock. Sustained-release formulation of prednisolone administered orally to man. J. Pharm. Sci. 60: 1028-1033 (1971).

4. J. G. Wagner. Biopharmaceutics and Relevant Pharmacokinetics, Drug Intelligence Publications, Hamilton, Ill., 1971, p. 112.

5. P. Finholt and S. Solvang. Dissolution kinetics of drugs in human gastric juice-the role of surface tension. J. Pharm. Sci. 57: 1322-1326 (1968).

6. W. A. Colburn and R. H. Buller. Radioimmunoassay for prednisolone. Steroids 21 : 833-846 (1973). 
7. Information Bulletin, Tritiated-Digoxin Radioimmunoassay Kit, Schwarz/Mann, Orangeburg, N.Y.

8. Guidelines for Biopharmaceutical Studies in Man, APhA Academy of Pharmaceutical Sciences, Washington, D.C., 1972, p. 2. 